\title{
Crecimiento, aceptación y tolerancia con una nueva fórmula láctea
}

\author{
Gloria Jury J.'; Carlos Castillo D. ${ }^{1,3}$; Eduardo Atalah S. ${ }^{2}$; \\ Rubén Puentes R. ${ }^{3}$; José Riumallo . $^{1}$
}

Modified cow milk formula: acceptability and infant's growth

\begin{abstract}
The purpose of this investigation was to evaluate the effect of a modified cow's milk formila on growth, tolerance and acceptability, which was studied in 2.097 infants selected from those controlled in primary health centers at Santiago. Chile: 1,119 were fed a modified cow's milk formula (LPM), while an unmodified powdered cow's milk (LP) was given to 978 controls of the same age and conditions, as well as solid foods after 4 months of age. Both experimental and control groups were followed arong a 4 months period for records of anthropametric data, morbidity, acceptability and tolerance for LF and LPM. We did not find sigrificant differerces in growth (values ranging 95 to $100 \%$ of NCHS standards W/A or H/Al. A slightly higher energy iritake and lower protein ntake was observed with LPM. Gastrointestinal symptoms were infrequently seen and of similar frequency and character in both 0 to 4 month old groups $(4.3 \%$ with. LPM vs. $5.3 \%$ with L.P at first control\}. These gastrointestira' signs were rnore frequently recorded inder LPM than with LP in the 8 to 12 months old group cnly at first control tcholics $8 \%$ vs. $4 \% \rho<0.01$ and abnormal stools $9.4 \%$ vs. $5.5 \% p<0.01$ ) Acute acceptability expresed as perceritage withdrawal from study was not significantly lower with LPM than LP (5 to $15 \%$ vs. $2 \%$ N.S.). The proposed modified formula seems to be a good alternative to powdered cow's milk for infants.

(Key words: milk, modified cow milk, infant nutrition, growth, acceptability.)
\end{abstract}

En Chile el Sistema Nacional de Servicios de Salud presenta una de las coberturas de atención más significativas de Latinoamérica, puesto que su infraestructura sanitaria le permite cubrir cerca de $70 \%$ de la población materno-infan$\mathrm{tij}^{1,2}$. Estudios nacionales han demostrado que al aumentar la cobertura disminuyen en forma importante la mortalidad infantil y la desnutri. ción. El número de controles de salud en menores de 6 años y embarazadas tambièn presenta en Chile una clara asociación con la cobertura del Plan Nacional de Alimentación Complementaria (PNAC) en dichos grupos ${ }^{3,4}$. Hasta 1974 el PNAC distribuía sólo leche de vaca en polvo con $12 \%$ de materia grasa ${ }^{5}$. A partir de entonces se inició una serie de modificaçiones tendientes a mejorar la efectividad del programa, destacando la incorporación de una leche de vaca en polvo con $26 \%$ de nateria grasa (LP); envases atractivos; la introducsión de estos productos en el

1. Instituto de Nutrición y Tecnología de los Alimentos, Universidad de Chile.

2. Departamento de Nutrición, Facultad de Medicina, Universidad de Clile.

3. Servicio de Pediatría. Hospital Sótero del Río. mercado abierto, eliminando la imagen de alimentos para pobres y la investigación de productos selectivos para los distintos grupos beneficiarios. Todo esto con miras a asegurar el máximo de eficacia y el mínimo de dilución intrafa. miliar ${ }^{6}$.

Por lo tanto, históricamente la entrega de leche entera en polvo ha representado un gran avance en la prevención de la desnutrición, entendido que la leche entera no modificada, por su alto contenido proteico y bajo contenido en hierro, ácjdos grasos esenciales, cobre y algunas vitaminas, no representa el alimento más adecuado para los lactantes menores de 1 afio $y$, más específicamente, los menores de 6 me$\operatorname{ses}^{2}, 8$

Teniendo en cuenta lo anterior, la unidad de bioquímica de los alimentos del INTA creó una förnula de leche de vaca en polvo, modificada de acuerdo a los requerimientos y recomendaciones del lactante menor (LPM), cuyas caracterís. ticas se presentaron con detalles en un artículo anterior'.

El propósito general de este estudio fue evaluar los efectos sobre el estado nutricional, tolerancia y aceptabilidad de una leche modifica- 
da (LPM), en un grupo representativo de lactantes sanos, controlados ambulatoriamente en los servicios de salad del área metropolitana, en comparación a un grupo semejante alimentado con leche de vaca en polvo (LP), con el fin de comprobar la posibilidad de incorporarla al PNAC.

\section{Material y Métodos}

La selección de la muestra y los procedimientos posteriores se efectuaron durante el control habitual do nitio sino, en siete consultorios de tres áreas de salud del Gran Santiago: Norte (consultorios Lucas Sierra, Quinta Buin y La Pincoya), Occidente (consultorios Santa Anita y Yazigi), Suroriente (consultorios La Granja y Alejandro del Ríor.

Los requisitos gener ales de ingreso fueron lactante sano, sin enfermedad ctónica o desnutrición aguda. pero de nacimiento mayor o igual a $2.000 \mathrm{~g} y$ parto no gemela:

Con el fin de estudiar la respuesta de los lactantes durante todo el ptimer año de vida, se establecieron tres colhortes de diferentes edades. En la cohorte I ingresaron 393 lactantes, menores de 2 meses 10 dias, que estuvieran recibiendo alimentación artificial exclusiva o alinentación mixta, minimo $150 \mathrm{ml}$ dia de leche no materna. Ellos fueron segujdos con controles mensuales hasta la edad de 4 meses (independiente del número de controles efectuados, que, según la edat de ingreso al estudio, variaron entre 2 y 4). En la cohorte II ingresaron 747 niños de 4 meses \pm 15 días, que fucron seguidos, como el tercer grupo, durante cuatro meses. A estos lactantes se les exigió, como condición adicional de ingreso, alimentación artificial exclusiva o mínimo $50 \%$ de sus necesidades lácteas djarias con leche no materna. En la fil cohorte ingresaron 957 lactantes de 8 meses \pm 15 dias de edad, que consumíar alimentación artificial exclusiva.

Una vez seleccionados, los 2.097 Jactantes fueron asignados en forma alcatoria, mediante un método estándar, a uno de los siguientes grupos: Experimental (GE), que recibió LPM, y Control (GC), constituido por aquellos que recibieron LP.

El aporte nutricional de las fórmulas utilizadas ture descrito en una publicación anterior9. LPM contiene, cada $100 \mathrm{~g}$ de polvo, $15 \mathrm{~g}$ de proteína, $26 \mathrm{~g}$ de grasa, $51 \mathrm{~g}$ de hidratos de carbono, $500 \mathrm{kcal} y 4 \%$ de las calorjas cono ácido linoleico. LP contiene $27 \mathrm{~g}$ de proteína, $26 \mathrm{~g}$ de grasa, $\mathbf{3 8} \mathrm{g}$ de hidratos de carbono, 496 kcal y $1,2 \%$ de las colorías como ácido tinoleico.

Dado que el producto experimental (LPM) no se encuentrà en el metcado, se decidió entregarlo en cantidades que permitieran cubrir las necesidades mensuales de consumo: $3 \mathrm{~kg}$ a los menores de un mes y a los lac tantes de 6 a 12 meses y $4 \mathrm{~kg}$ a aquellos de 1 a 5 meses. La alimentación del grupo control se hízo en base a LP, entregándola en las cantidades establecidas por el Ministerio de Salud de Chile ( $2 \mathrm{~kg}$ mensuales).

Las indicaciones de alimentación de los lactantes de ambos grupos se hicieron según una pauta estándar por edades, especiaimente diseñada para este efecto.
En los menores de 4 meses se indicó LPM diluida al I5\%, sin agregado de azúcar ni aceite (LPM $1=75,0$ $\mathrm{kcal} / \mathrm{L00} \mathrm{ml} ; 2,2 \mathrm{~g}$ prot/100 ml). Desde los 4 meses, lasta el año de vida, se indicó LPM diluida al $12,5 \%$ adicionada de un $5 \%$ de azucar o harina (LPM $2=82,4$ $\mathrm{kcal} / 100 \mathrm{ml} ; 1,9 \mathrm{~g}$ prot/100 ml). La pauta de indica. ciones con LP se hizo respetando las normas vigentes: 0 a 3 meses 29 dias, LP diluida al $10 \%$, más $5 \%$ de azúcar (LP $1=69,5 \mathrm{keal}$ y $2,7 \mathrm{~g}$ proteína $/ 100 \mathrm{ml}$ ) $y$ desde los 4 meses hasta el año de vida se indícó al $10 \%$ más $5 \%$ de azúcar 3 $5 \%$ de harina $(\mathbf{L P} 2=$ $86,7 \mathrm{k}$ cal y 3,1 g proteína $100 \mathrm{ml}$ ). La introducción de alimentos sólidos se prescribió a los 4 meses de edad, comenzando en forma progresiva con sopa-puré de verduras (verduras, cereal y carne o pollo) y una fruta, introduciendo luego, hasta el año de vida, mayor variedad de alimentos, adecuando la consistencia a las nece. sidades del lactante.

Las madres recibieron indicaciones escritas sobre la preparación de mamaderas, así como una medida de S g y un biberón graduado de $250 \mathrm{ml}$, con el fin de estandarizar la ingesta.

Los niños fueron controlados por profìsionales caljficados. Se efectuaron registros mensuales de peso $y$ talla, con técnicas e instruntental estándar a lo latgo de cuatro meses en los grupos etarios II y III y hasta e] cuarto mes de vida en el grupo I. En cada uno de los controles la madre respondió una encuesta de aceptabilidad del producto a mediano y latgo plazo por recordatorio de 24 horas, estimando la telación cantidad ingerida/cantidad ofrecida por día.

La aceptabilidad fue registrada en términos del porcentaje de lactantes que permaneció en el estudio (ingresados-xcluidos/ingresados) $\times 100$ ).

La tolerancia fue medida en términos de aparición o ausencia de síntomas gastrointestinales segin recordatorio de siete días. Durante cada control se cntregaron pautas de alimentación escritas y se hizo refuerzo educativo sobre la importancia de la alimentación e higiene del niño, junto con las actividades habituales del control de nirio samo.

\section{Resultados}

La asignación aleatoria a los grupos experimental y control resultó en grupos plenamente comparables, en cada cohorte etaria, en cuanto a peso y talla de nacimiento, edad gestacional, edad de las madres y número de hermanos (tabla 1).

En la figura 1 se muestra la evolución de las relaciones peso/edad (P/E) y peso/talla $(\mathrm{P} / \mathrm{T})$, establecidos por comparación con el percentil 50 del estándar del Centro de Estadísticas de Salud de E.U.A. (NCHS) para la edad o talla correspondiente. Las relaciones $\mathrm{P} / \mathrm{E}$ y $\mathrm{P} / \mathrm{T}$ son absolutamente comparables al ingreso y en cada uno de los controles en cada grupo etario, man. teniéndose dentro de límites normales durante 
Tabla 1

Características generales grupo experimental y control por grupo etazio

\begin{tabular}{|c|c|c|c|c|c|c|}
\hline \multirow{2}{*}{$\begin{array}{l}\text { Edad } \\
\text { Grupo }\end{array}$} & \multicolumn{2}{|c|}{$0-4$ meses } & \multicolumn{2}{|c|}{$4-8$ meses } & \multicolumn{2}{|c|}{$8-12$ meses } \\
\hline & $\mathrm{GE}$ & GC & GE & GC & $\mathrm{GE}$ & GC \\
\hline $\mathbf{N}$ & 213 & 180 & 398 & 349 & 508 & 449 \\
\hline $\mathrm{M} / \mathrm{F}$ & 1,07 & 1,20 & 1,10 & 0,98 & 1,05 & 3.05 \\
\hline TN.(g) & $3,2 \pm 0,45$ & $3,2 \pm 0,49$ & $3,2 \pm 0,45$ & $3,2 \pm 0,44$ & $3,3 \pm 0,45$ & $3,3 \pm 0,4$ \\
\hline TN.(cm) & $49,0 \pm 2,0$ & $49,3 \pm 2,0$ & $49,4 \pm 1,9$ & $49,4 \pm 2,1$ & $49,6 \pm 1,9$ & $49,7 \pm 1,8$ \\
\hline E. Gest. (sem) & $39,0 \pm 1,6$ & $39,0 \pm 1,4$ & $39,2 \pm 1,4$ & $39,2 \pm 1,4$ & $39,3 \pm 1,4$ & $39,3 \pm 1,2$ \\
\hline Edad Madre & $26,7 \pm 7,1$ & $25,5 \pm 6,3$ & $25,7 \pm 5,7$ & $26,3 \pm 6,0$ & $26,2 \pm 6,2$ & $25,6 \pm 5,7$ \\
\hline No hijos & $2,4 \pm 1,7$ & $2,3 \pm 1,6$ & $2.3 \pm 1,6$ & $2,2 \pm 1,4$ & $2,1 \pm 1,4$ & $2,1 \pm 1,3$ \\
\hline
\end{tabular}

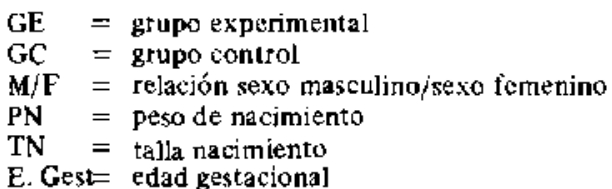

RELACIONES PIE $\times \mathrm{P} / \mathrm{T}$ I\% del eslandor NCHS

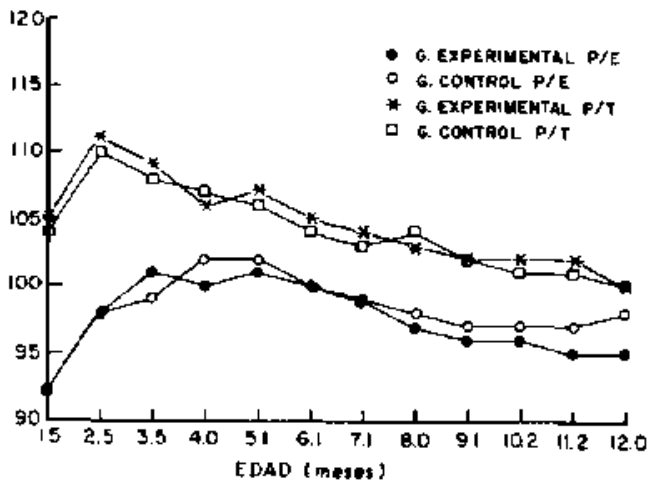

Fig. 1: Erolución de los parámetros antropométricos: peso para la edad y peso para la talla de los grupos aljmentados con LPM (G. Experimental) y con LP (G. Control) durante el estudjo. Las edades indicadas coresponden al promedio de edades de ambos grupos en el momento del control.

todo el estudio. Dentro de díchos límites, llama la atención, en ambos grupos (GE: LPM y GC: LP), la tendencia de los dos indicadores a mejorar durante los primeros meses de vida $y$ a deteriorarse discretamente a partir de los 5 meses de edad.

En la figura 2 se describe el incremento mensual de peso de los niflos con LPM y LP. No se observaron diferencias significativas o biológica. mente importantes entre ambos, siendo los incrementos comparables a los promedios nomales del estándar NCHS.

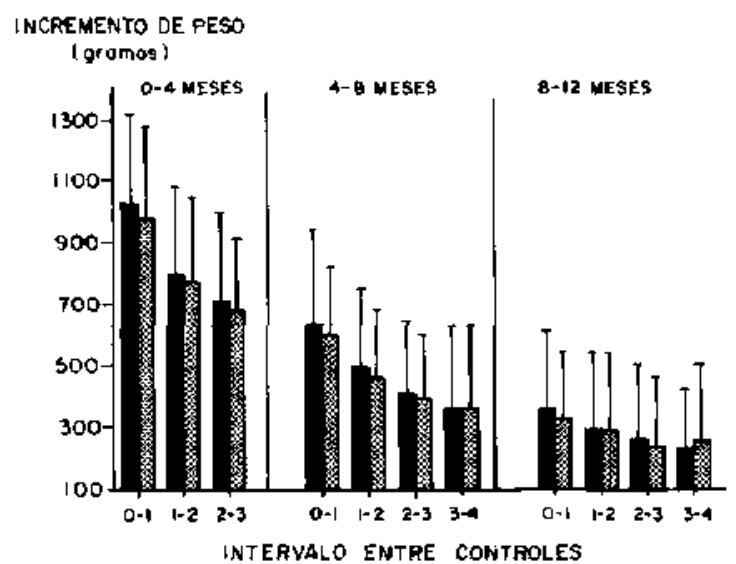

Fig. 2: Incremento promedio mensual de peso (g) de los grupos alimentados con LPM (G. Experimental) y con LP (G. Control). La evaluación está medida como el incremento promedio de peso experimentado de un control a otro en las tres cohortes estudiadas.

Los incrementos promedio mensuales de talla tampoco mostraron diferencias significativas $y$ fueron similares a los calculados para el percentil 50 de las tablas del NC.HS. En los niños de 0 a 4 meses el incremento promedio de talla varió entre 3,2 y $2,1 \mathrm{~cm} \cdot$ mes. En los de 4 a 8 meses entre 2,3 y $1,4 \mathrm{~cm} \cdot$ mes y en $\operatorname{los}$ de 8 a 12 meses entre 1,5 y $1,3 \mathrm{~cm} \cdot$ mes.

El aporte energético proporcionado por las fórmulas LPM y LP a los niños de 0 a 4 meses cubrió entre 82 y $93 \%$ de sus requerimientos diarios, debido a la persistencia de algún grado 
de lactancia materna. En cambio, en los dos grupus mayores las diferencias observadas fueron atribuibles a la introducción paulatina de aljmentos sólidos $(90$ a $66 \%$ en lactantes de 4 a 8 meses y 63 a $51 \%$ en los de 8 a 12 meses). Considerando sólo el aporte energético de las fómulas, se observaron diferencias estadisticamente significativas en favor del grupo experimental, en tramos etarios de 4 a 8 y 8 a 12 neses con respecto al grupo control.

$\mathrm{La}$ ingestión de proteínas, debido a su contenido en las fómulas utilizadas, resultó significativamente mayor en el grupo control para todas las cohortes etarias (tabla 2).

La tolerancia de la LPM incluyó el registro de la frecuencia de aparición de síntomas como cólicos, deposiciones alteradas (líquidas o dis. gregadas), constipación y vómitos. En el primero y segundo grupo etario la frecuencia de aparición de síntomas gastrointestinales, según la madre, fue similar en ambos grupos (LPM y LP), durante los cuatro controles efectuados. La frecuencia de cólicos en el primer control en el tramo de 0 a 4 meses fue $11 \%$ y $8,4 \%$ en lactantes con LPM y Ll', respectivamente. En el segun. do control, las correspondientes frecuencias fueron $1,6 \%$ y $1 \%$. En los nitios de 4 a 8 meses la frecuencia de dicho signo en el primer control fue 4,7\% y $5,3 \%$ en ambos casos. En cambio para el grupo entre 8 y 12 meses de edad, en el primer control la frecuencia de cólicos había sido $8 \%$ con LPM (GE) y $4 \%$ con LP (GC): deposiciones líquidas $9,4 \%$ (GE) vs. 5,5 (GC); constipación $10,6 \%$ (GE) vs: $6,2 \%$ (GC) y vó. mitos $12,7 \%$ (GE) vs. $7,8 \%$ (GC); en otras palabras, significativamente mayor $(\mathrm{p}<0,001$ ) para el grupo que recibió LPM. Sin embargo, en los controles posteriores la frecuencia de aparición de sintomas gastrointestinales resultó semejante en ambos grupos.

Con LP, la accptabilidad aguda en cada cohorte fue superior a $98 \%$. Con LPM la aceptabilidad fue de $92 \%, 89 \%$ y $85 \%$ en los tramos etarios I. II y III, respectivamente.

La aceptabilidad a mediano y largo plazo fue muy semejante para los grupos control y expe. rimental en cada cohorte etaria. En la primera cohorte, la aceptabilidad varió entre 96 y $98 \%$ para LPM y 95 a $98 \%$ para LP. En los niños de 4 a 8 meses $95 \%$, pronedio, en cada uno de los controles del GE (LPM) y entre 95 y $96 \%$ para el (jC (LP). En el tramo de 8 a 12 meses la aceptabilidad varió entre 91 y $95 \%$ para LPM, 93 y 95\% para $\mathrm{LP}$

\section{Discusión}

De acuerdo a los resultados presentados, la leche nodificada (LPM) es capaz de sostener un crecimiento normal, semejante al obtenido con la fórmula en uso, durante el primer año de vida. Esto se corroboró con incrementos de peso promedio, en los primeros meses de vida, superiores a los del percentil 50 de las tablas NCHS $^{10,11}$. La tendencia a superar la norma, disminuye a medida que avanza la edad, haciéndose levemente inferior a partir del quinto mes de vida $y$ manteniéndose así hasta el año de edad, tanto con LPM como la misma situación con $\mathrm{LP}$, en coincidencia con otros estudios nacionales, en los que se ha atribuido ește descenso a la introducción de alimentación no láctea o a la importante disminución de la lactancia materna en esta edad ${ }^{12}$. En lactantes chilenos alimentados en forma exclusiya al pe-

\section{Tabla 2}

lngesta protejca (g $\cdot \mathrm{kg} \cdot \mathrm{dja})$ grupo experimental y g̣upo control durante el per jodo de observación

\begin{tabular}{|c|c|c|c|c|c|c|}
\hline \multirow{2}{*}{$\begin{array}{l}\text { Eáad } \\
\text { Grupo }\end{array}$} & \multicolumn{2}{|c|}{$0-4$ meses } & \multicolumn{2}{|c|}{4 - 8 meses } & \multicolumn{2}{|c|}{$8-12$ meses } \\
\hline & GE & $\mathrm{GC}$ & GE: & $\mathrm{GC}$ & $\mathrm{GE}$ & GC \\
\hline Ier. Control & $2,8 \pm 1,0$ & * $3,5 \pm 1,5$ & $2.4 \pm 0,8$ & * $3,0 \pm 1,3$ & $1,7 \pm 0,6$ & $* 2,3 \pm 0,8$ \\
\hline $2^{\circ}$ Control & $2,7 \pm 0,9$ & $* 3,5 \pm 1,4$ & $2,1 \pm 0,7$ & $=2,9 \pm 1,0$ & $1,7 \pm 0,5$ & - $2,3 \pm 0,7$ \\
\hline $3^{e r}$ Control & $2,5 \pm 0,8$ & * $\quad 3,3 \pm 1,4$ & $2,0 \pm 0,7$ & * $2,6 \pm 0,9$ & $1,7 \pm 0,5$ & $-2,2 \pm 0,7$ \\
\hline $4^{\circ}$ Control & $2,4 \pm 0,9$ & * $3,1 \pm 0,9$ & $1,9 \pm 0,7$ & $\neq 2,5 \pm 0,8$ & $1,7 \pm 0,5$ & * $2,1 \pm 0,6$ \\
\hline
\end{tabular}

\footnotetext{
$\mathrm{GE}=$ grupo experimental

$\mathrm{GC}=$ grupo control

* $\quad=\mathrm{p}<0,001$
} 
cho, se ha descrito un crecimiento semejante al observado en este estudio hasta el quinto mes de vida, levemente mayor entre el quinto y octavo mes y nuevamente comparable hasta el año de edad ${ }^{13}$.

En cuanto a la talla, su incremento promedio con LPM y LP fue muy similar al calculado para el percentil 50 de las tablas de crecimiento NCHS. La mantención del incremento normal de talla, unido a la disminución de la velocidad de ascenso ponderal, a partir del quimto mes de vida, explican el leve deterioro de las relaciones $\mathrm{P} / \mathrm{E}$ y $\mathrm{P} / \mathrm{T}$ observadas en ambos grupos ${ }^{14}$.

La cantidad de LPM entregada a las madres, en este estudio, y sur forma de preparación, fueron calculadas para cubrir el total de los requerimientos calóricos hasta el cuartu thes de vida, momento en que se prescribió el injcio de la alimentación no láctea ${ }^{15-17}$. La entrega de $2 \mathrm{~kg}$ de LP, norma actual del PNAC, teórica. mente cubre sólo $68 \%$ de los requerimientos energeticos del mes de edad y $50 \%$ de ellos a los 6 meses. Por toda esta razón se entregaron $4 \mathrm{~kg}$ de LPM a los niños de 2 a 6 meses. La cobertura de los requerimientos calóricos con LPM es necesaria para que se cumpla una de las ventajas comparativas de este producto sobre la $L P$, cual es la de cubrir totalmente los requerimientos de ácjdos grasos esenciales, vitaminas y minerales ${ }^{18}$.

La práctica habitual es indicar dos comidas a los lactantes de 6 a 8 meses, las cuales representan un porcentaje muy variable de los requerimientos calúricos proteicos, según la situación socioeconómica y cultural de la madre. Entre los 6 y 12 meses los niños reciben alrededor de 750 $\mathrm{ml}$ diarios de fórmula láctea $\mathrm{y}$ es importante que ella cubra al menos 50 ó $60 \%$ de los requerimien. tos energéticos y todos los requerimientos proteicos, considerando que estos últimos pueden estar aumentados en lactantes de bajo nivel socioeconónico, debido a factores ambientales ${ }^{14}, 19$. La cantidad de leche modificada (LPM) eлtregada en este rango de edad $(3 \mathrm{~kg})$ permitió, en teoría, cubrir aproximadamente $87 \%$ de los requerimientos calóricos medios a los 6 meses y $61 \%$ a los 12 meses de edad, con la sola adición a la fórmula de $5 \%$ de azúcar. $\mathrm{La}$ cobertura de esta fómula para los extremos superiores del rango sería de $69 \%$ a los 6 meses $y$ de $50 \%$ a los 12 meses.

En los analisis de ingesta real, la fórmula con LPM ofrece un aporte calórico medio de $90 \%$ o más de los requerimientos hasta los 5 a 6 meses de edad, pero luego cae progresivamente a 70 u $80 \%$ de los requerimientos hasta los 8 meses $y$ a alrededor de $60 \%$ a los 8 a 12 meses de vida, porcentajes significativamente superiores a los observados con LP (aproximadamente 90\% para los primeros 4 meses de vida, 50 y $60 \%$ entre los 8 y 12 meses de edad). Este hecho puede ser debido, en parte, a la mayor disponibilidad de LPM por parte de la madre en todas las cohortes etarias.

Por lo tanto, las formulaciones con LPM 15\% sin azúcar para lactantes de hasta 4 meses $y$ $12,5 \%$ más $5 \%$ de azúcar para lactantes mayores de 4 meses presentan ventajas desde el punto de vista calórico, con respecto a la fórmula de LP utilizada en dichas edades.

Uno de los problemas de las fómulas con leche de vaca empleadas en la alimentación de lactantes ha sido su alta concentración proteica. Esto produce ingestiones de proteina que exceden lo recomendado, aun tomando en cuenta posibles déficit de la alimentación no láctea, en los casos que corresponde ${ }^{20}$. En el presente estudio, la ingesta proteica para los tres grupos etarios y en cada uno de los controles fue significativamente superior con LP que con LPM. Esto implica que la carga nitro. genada es menor con LPM, lo que puede ser favorable para el crecimiento y función renal. especialmente en lactantes menores ${ }^{21,22}$.

La tolerancia de LPM, medida como la frecuencia de aparición de sintomas gastrointestinales, fue adecuada y plenamente comparable a la observada con LP en los grupos de lactantes menores, mientras que los lactantes mayores ( 8 a 12 meses) alimentados con LPM presentaron una mayor frecuencia de síntomas en las etapas iniciales de la introducción del producto. Estos sintomas, posiblemente, responsables de la de. serción del estudio de algunos casos, podrían deberse a que se trataba de niños acostumbrados a fórmulas en base a LP, bruscamente cambiados a LPM de diferente sabor, textura y composición química.

Este hecho se refleja, ademas, en la aceptabili. dad aguda, medida como el porcentaje de lactantes que permaneció en el estudio, donde se observa que a medida que aumenta ta edad el porcentaje de lactantes que abandona el estudio es mayor $(8 \%, 11 \%$ y $15 \%$ para cada cohorte). En cambio, cuando se midió lo realmente ingerido, expresado como porcentaje del volumen 
de fórmula ofrecida al lactante, la aceptabilidad de la $L P M$, en los casos que permanecieron en el estudio, fue similar para el grupo control y experimental. Estas cifras son comparables a las encontradas para la LP, pero mayores que los de otras fómulas ensayadas ${ }^{23}$.

Por lo tanto, frente a la eventual introducción de LPM en el PNAC, sería aconsejable realizaria en edades tempranas como fórmula inicial, en el momento que el niño pasa de alimentación natural a alimentación artificial o mixta, a fin de minimizar la aparición de este tipo de síntomas.

La posibilidad de entregar a temprana edad una fórmula láctea sin agregados representa ventajas, pues al no requerir otros ingredientes resulta económica, se facilita su preparación $y$, potencialmente, se reduce el riesgo de contaminación e infección, debido a menor manipulación del producto.

En concordancía con los antecedentes presentados. la fỏrmula evaluada (LPM) es una buena alternativa en la alimentación de lactantes meno. res de un año y podría ser considerada en el plan nacional de alimentación complementaria.

\section{Resumen}

Se evaluó, en forma controlada, los efectos sobre el estado nutricional, tolerancia y aceptabilidad de una leche modificada (LPM), en un grupo representativo de lactantes sanos, contro. lados en los servicios de salud del Area Metropolitana, pareados a un grupo semejante, alimentado con una leche de vaca en polvo (LP), con el fin de controlar la posibilidad de incorporarla al PNAC. Del total de lactantes $(2.097)$, 393 lactantes entre 0 y 4 meses de edad, 747 entre 4 y 8 meses $y 957$ entre 8 y 12 meses. Todos fueron seguidos durante 4 meses con controles antropométricos, de morbilidad, aceptabilidad y tolerancia de la fórmula. Los resultados no mostraton diferencias significativas en crecimiento medido por $\mathrm{P} / \mathrm{E}$ o $\mathrm{P} / \mathrm{T}$ (valores entre 95 y $100 \%$ de los estándares NCHS). Se vio una leve mayor ingesta de energía y menor de proteúnas con LPM. La aparición de signos gastrointestinales fue baja y comparable entre los dos grupos, tanto en la primera cohorte etaria (cólicos $11 \%$ con LPM y $8 \%$ con LP al primer control; 1,6 vs. $1,0 \%$ al segundo control) como en la segunda cohorte etaria $(4,3 \%$ con LPM vs.
$5,3 \%$ con L.P al primer control); en cambio, durante el primer control, el grupo de 8 a 12 meses alimentado con LPM presentó mayor incidencia de síntomas que GC (cólicos $8 \%$ vs. $4 \%$; deposiciones alteradas $9,4 \%$ vs. $5,5 \%$, $\mathrm{p}<0,001$ ); durante los 3 controles posteriores la diferencia no fue significativa. La aceptabilidad aguda, medida como porcentaje de exclusiones de niños del estudio. fue más baja con LPM que con LP (5 a $15 \%$ vs. $2 \%$, N.S.). Se concluye que la fómula estudiada es una buena alterna. tiva de alimentación láctea en menores de 1 año y que podria ser incorporada al Programa de Alimentación Complementaria.

(Palabras clave: leche de vaca modificada, alimentación de lactantes, tolerancia, aceptación, resultados nutricionales.)

\section{Agradecimientos}

Nuestros sinceros agradecimientos a todo el personal de los consultorios Yazigi, Santa Anita, Lucas Sierra, Quinta Buin, La Pincoya, La Granja y Alejandro del Río, quienes participaron con interés y excelencia en cada etapa del presente proyecto.

\section{Referencias}

1. González, N.: Intante, B.: Programas de alimentación complementaria del sector galud en Chile. Bol of Sanit Panam 1980; B9: 6-10.

2. Cruzat, A.; González, N.: Mordones, S.F.: Moenne. M.: Sanchez, H.: Cobertura de los programas nutricionales $y$ de salud en los sectores de menores ingresus. Rev Méd Chile 1982; $110 \div 585.592$.

3. Atalah, E: Puentes. R.i Costillo, D.C.; Radrigán, M.E.: Programa Nacional de Alimentación Complementaria. 1965-1985. Rey Chil Pedjatr. 1985; 56: $362-368$.

4. Mardones, R.F.: Cobertura del PNAC y tasas de desnutrición en Chiłe. 1982-1983. Rev Chíl Nutr 198:; $1: 54-55$.

5. Conzólez, N.: Programas de alimentación cumplementaria PNAC del Ministezio de Salud-Chile, Documento de trabajo, Santiago, octubre, 1982.

6. Mardones, F.: González, N.: Mardones, R.F., Salinas, J.; Albala, C.: Programa Nacional de Alimentación Complementaria en Chile en el período 1937-1982. Rev Chil Nutt 1986; 3: 173. 182.

7. ESPGAN Commitee on nutrition: Gujdelines on infant nutrition. I Recomendations for the conposition of an adapted formula. Acta Paediatr Scand 1977 Suppl 262: 1-63.

8. ESP(;AN Commitce on nutrition: Guidelines on infant nutrition. Il Recomendations tor the composition of follow-up formula and beikast. Acta Paediatr Scand 1981; Suppl 287: 1-25. 
9. King, J.; De Pablo, S.; Montes de Oca, F.: Uauy. $R$.: Formulación, fabricación y Evaluación de una fórmula infantil para el PNAC en Chile. Rev Chil Pedjatr (en prensa).

10. U.S. Departmen of Health, Education, and Welfare. Public Hedith Service of Nationat Center for Health Statistics: NCHS Grouth Curves for children. Birth-18 years. L'nited States. Series 11 , Number 165. Nov. 1977.

11. FAO/OMS/UNU: Necesidades de energia y protei. nas. Informe de una reunión consultiva conjunta FAO/OMS/UNU de expertos. OMS, Ginebra. Serie de informes técnicos 724,1985 .

12. Lopez, I, Cabiol, C.; Arruch, $S_{-i}$ Rilera, E.: Vargas, $S .:$ Lactancia materna y aumento de peso en el primer año de vida, Rev Chil Pediatr 1980; 51 : $473-479$.

13. Juez, $G_{\text {.; }}$ Diaz. S.l.; Peralta. O.M.: Croxatto. $H B$.: Lactancia materna exclusiva: Crecimiento del lactante en un grupo seleccionado de nizios chilenos. Rev Chil Pediatr 1984; 55: 225-230.

14. Jordan, J.: El lactante de 0-2 años: Antropome tría y crecimiento. En: Crecimiento y Desarrollo. Hechos y Tendencias. Eds. Cusminsky, M.: Moreno, E.; Ojeda, E. Pub Cientificas 510. OPS-OMS 1988.

15. Rama de Nutrición: Alimentación normal del lactante. Recomendaciones de la Rama de Nutrición de hacjedad Chilena de Pediatría. Rev Chil Pediatr 1988; 59: $139-143$.

16. American Academy of Pediatrics: Commitee on Nutrition. The use of whole cow's milk in infancy. Pediatrics 1983; $72: 253-255$.
17. Abejá, E.: Nuevas tendencias en la alimentación del niño menor de dos años: su relación con la nutrición $y$ el recimiento. En: Crecimiento $y$ Desarrollo. Hechos y Tendencias. Eds. Cusminsky. M.; Moreno, E.,; Ojeda, E. Pub científicas 510 . OPSAMS 1988.

18. Ueuy. R.; Jury, G; Ruimallo, J.: Castillo-Durén. C.: Evaluación nutricional $y$ control de calidad leche Purita Modificada. Proyecto presentado al Ministerio de Sa]ud de Chile. INTA 1984.

19. Andrade, M.; Aralah, E.; Rojas. J.: Taibo, M.: Alimentación del lactante $y$ pteescolar. En Nutrición. Prevención de riesgos y tratamiento dietético. Eds. Olivares, S.; Soto, D.: Zacarías, I. CONFELANYD. Chjle, 1989.

20. Ruimalio, J.: Castillo.Durán, C.; Jury. G.: Evaluación nutricional y control de calidad leche Purita Modificada. Informe final, tomos I, II $y$ III presentados al Ministerio de Salud de Chile. INTA 1986.

21. Fomon, S.J. Filer, L.F.; Anderson, T.A.; Ziegler. E. $E_{1}$ : Recommendation for focding nomal infants. Pediatrics $1979 ; 63: 52-54$.

22. Fomon, S.J.: thfant Nutrition 2nd ed. Philadelphja, WB Saunders Company, 1974.

23. Stekel. A.: Pizarro, F.: Olivares. M.: Chadrid, P.: Llogumo, S.: Cavazzo. H.: Hertrampf. E.; Walter, $T$. Prevention of deficiency by milk fortification. 1II. Effectiveness under the usual operational tonditiones of a nation - wide food program. Nutrition Report International 1988: 38: 119128 .

\section{AVISO A LOS AUTORES}

Por acuerdo del Comité Editorial, la Revista Chilena de Pediatria devolverá sin tramitar todos los trabajos que no den estricto cumplimiento al Reglamento de Publicaciones, que se edita en cada número, y a las Instrucciones a los Autores, que aparecen en los números 3 y 6 de cada volumen desde 1986. 